\title{
Absolute motions of globular clusters
}

\section{HST astrometry and VLT radial velocities in NGC $6397^{\star}$}

\author{
A. P. Milone ${ }^{1}$, S. Villanova ${ }^{1}$, L. R. Bedin ${ }^{2}$, G. Piotto ${ }^{1}$, G. Carraro ${ }^{1,3}$, J. Anderson ${ }^{4}$, I. R. King ${ }^{5}$, and S. Zaggia ${ }^{6}$ \\ 1 Dip. di Astronomia, Univ. degli studi di Padova, vic. Osservatorio 2, 35122 Padova, Italy \\ e-mail: milone-villanova-piotto@pd.astro.it \\ 2 European Southern Observatory, Karl-Schwarzschild-Str. 2, 85748 Garching, Germany \\ e-mail: 1bedin@eso.org \\ 3 Andes Fellow, Departamento de Astronómia, Universidad de Chile, Casilla 36-d, Santiago, Chile \\ e-mail: gcarraro@das.uchile.cl \\ 4 Dept. of Physics and Astronomy, Mail Stop 108, Rice University, 6100 Main Street, Houston, TX 77005, USA \\ e-mail: jay@eeyore.rice.edu \\ 5 Dept. of Astronomy, Univ. of Washington, Box 351580, Seattle, WA 98195-1580, USA \\ e-mail: king@astro.washington.edu \\ 6 INAF - Osservatorio Astronomico di Trieste, via Tiepolo 11, 34131 Trieste, Italy \\ e-mail: zaggia@ts.astro.it
}

Received 3 February 2006 / Accepted 27 April 2006

\section{ABSTRACT}

\begin{abstract}
In this paper we present a new, accurate determination of the three components of the absolute space velocity of the Galactic globular cluster NGC $6397\left(l \simeq 338^{\circ}, b \simeq-12^{\circ}\right)$. We used three HST/WFPC2 fields with multi-epoch observations to obtain astrometric measurements of objects in three different fields in this cluster. The identification of 33 background galaxies with sharp nuclei allowed us to determine an absolute reference point and measure the absolute proper motion of the cluster. The third component was obtained from radial velocities measured from spectra from the multi-fiber spectrograph FLAMES at UT2-VLT. We find $\left(\mu_{\alpha} \cos \delta, \mu_{\delta}\right)_{\mathrm{J} 2000.0}=$ $(+3.39 \pm 0.15,-17.55 \pm 0.15)$ mas yr$^{-1}$ and $V_{\text {rad }}=+18.36 \pm 0.09( \pm 0.10) \mathrm{km} \mathrm{s}^{-1}$. Assuming a Galactic potential, we calculate the cluster orbit for various assumed distances and briefly discuss the implications.
\end{abstract}

Key words. astrometry - globular clusters: individual: NGC 6397 - techniques: spectroscopic - Galaxy: kinematics and dynamics

\section{Introduction}

After 15 years of activity, the Hubble Space Telescope (HST) is producing large volumes of accurate astrometry from the measurements that the high spatial resolution of WFPC2 and ACS images allows. In the archive are numerous fields with multi-epoch observations that are well separated in time, and first-epoch observations exist for even more fields. These are a gold mine for proper-motion measurements in a large variety of Galactic and extragalactic fields, including the crowded regions of globular clusters (GCs).

Globular clusters are important Galactic tracers; their orbital motion offers insight into the kinematics and dynamics of the halo, and their motion provides important clues to the distribution of mass in the Galaxy. Moreover, knowledge of GC orbits enables us to study their interaction with the Galactic disk and halo and is important to dynamical studies of the relationship of a cluster present-day mass function to the initial one.

In the first paper of this series (Bedin et al. 2003, Paper I), we presented the absolute proper motion of the globular cluster M4, using a background QSO as reference. In this

* Based on observations with the NASA/ESA Hubble Space Telescope, obtained at the Space Telescope Science Institute, which is operated by AURA, Inc., under NASA contract NAS 5-26555, and with the ESO Very Large Telescope + FLAMES at the Paranal Observatory, Chile, under the program 71.D-0076. paper we use the background galaxies in the field of another nearby GC, NGC 6397. We made use of archival material, as well as of new images taken as a second epoch (GO-8656). We also used ground-based spectra from the multi-fiber spectrograph FLAMES (Pasquini et al. 2002) on UT2-VLT to measure the radial velocity of the cluster. Finally, with the new measurements of proper motion and radial velocity, and an assumed Galactic potential, we explore the orbit of the cluster.

\section{Observations, data reduction}

\subsection{WFPC2/HST: proper motions}

In Fig. 1 we use equatorial coordinates to show the footprints of the three WFPC2 fields of NGC 6397 that we used in the present work. The center of the figure coincides with the cluster center. In the following we refer to each field as B, C, and D, as labeled in the figure. Field A was not used in the present work because the two epochs were oriented differently, and this would have required a different approach to the reduction, since the chips can no longer be treated independently.

Table 1 lists for our three fields the exposures that we used. Almost all of the image sets are dithered well, with both large and fractional pixel offsets, allowing precise astrometric measurements and a more accurate assessment of the errors. 


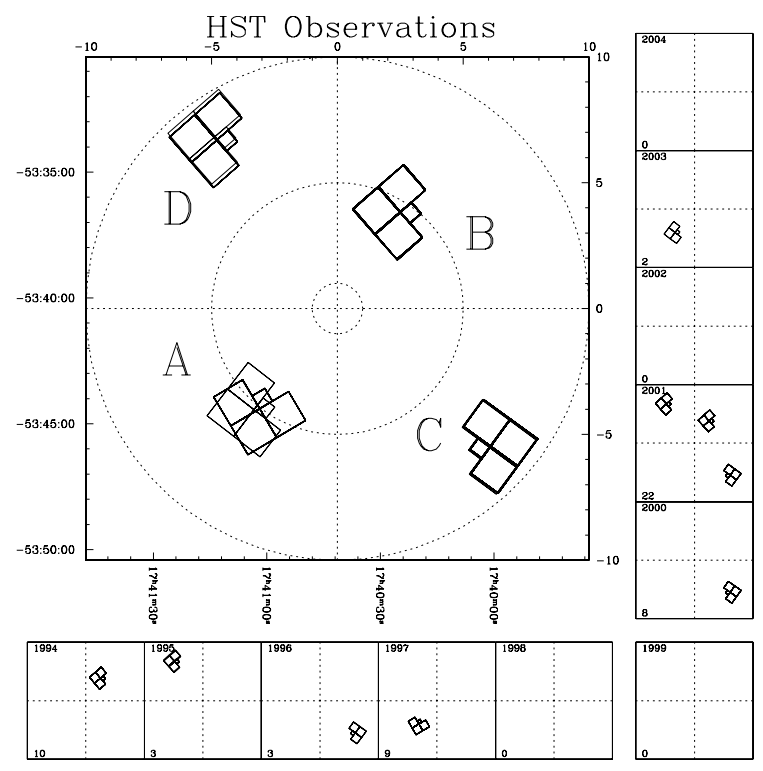

Fig. 1. Finding chart of the WFPC2 fields considered for this work. The region is $20^{\prime} \times 20^{\prime}$ wide and is centered on the center of NGC 6397 . The fields measured in this work are labeled "B","C", and "D". For completeness we also show field " $A$ ", which we did not use here (see text) but will use in forthcoming papers. Fields A, B, C, D are centered 5', $4.5,8^{\prime}$, and $8^{\prime}$ from the cluster center, respectively.

Table 1. Description of the data set used in this work.

\begin{tabular}{|c|c|c|c|c|}
\hline$\overline{\mathrm{IID}}$ & date & EXP-TIME & $\overline{\text { FILT }}$ & $\overline{\overline{\mathrm{GO}}}$ \\
\hline \multicolumn{5}{|c|}{ first epoch } \\
\hline $\mathrm{A}$ & Apr'97 & $9 \times \sim 800 \mathrm{~s}$ & $F 814 W$ & 6797 \\
\hline \multirow[t]{2}{*}{$\mathrm{B}$} & Mar'94 & $3 \times \sim 1000 s+2100 s+500 s$ & F814W & 5092 \\
\hline & Mar'94 & $5 \times \sim 1000 \mathrm{~s}+400 \mathrm{~s}$ & F606W & 5092 \\
\hline \multirow[t]{4}{*}{$\mathrm{C}$} & Oct'96 & $3000 s+2 \times 1900 s$ & F814W & 6802 \\
\hline & Oct'96 & $1300 s+700 s$ & $F 814 W$ & 7203 \\
\hline & Oct'96 & $1700 s+400 s$ & $F 606 W$ & 6802 \\
\hline & Oct'96 & $300 \mathrm{~s}$ & F606W & 7203 \\
\hline \multirow[t]{3}{*}{$\mathrm{D}$} & Apr'95 & $1800 \mathrm{~s}$ & $F 814 W$ & 5369 \\
\hline & Apr'95 & $2 \times 1200 \mathrm{~s}$ & $F 814 W$ & 5370 \\
\hline & \multicolumn{4}{|c|}{ later epochs } \\
\hline $\mathrm{A}$ & Mar'03 & $500 s+260 s$ & F606W & 9676 \\
\hline \multirow[t]{2}{*}{ B } & Apr'01 & $3 \times 1300 s+1200 s$ & $F 814 W$ & 8656 \\
\hline & Apr'01 & $4 \times 1300 \mathrm{~s}$ & F606W & 8656 \\
\hline \multirow[t]{2}{*}{$\mathrm{C}$} & Oct'00 & $1200 s+7 \times 1300 s$ & F606W & 8656 \\
\hline & Oct'01 & $1200 s+5 \times 1300 s$ & $F 814 W$ & 8656 \\
\hline $\mathrm{D}$ & Apr'01 & $2 \times 1300 \mathrm{~s}$ & $F 814 W$ & 8656 \\
\hline
\end{tabular}

Positions were measured for each filter, chip, and epoch independently by using the effective-point-spread-function (ePSF) algorithms described by Anderson \& King (2000). As in Paper I, for proper-motion measurements we used local transformations, and the best distortion corrections (Anderson \& King 2003), after removing the 34th-row error (Anderson \& King 1999). The photometry was calibrated to the Vega-mag flight system, following the recipe of Holtzman et al. (1995). For stars with $>100 \mathrm{DN}$ in their brightest pixel, we were able to measure positions in a single image with an error of $<0.04$ pixel in each coordinate.

In order to avoid introducing systematic errors, we measured the proper motions from two epochs in the same filter. When two epochs were available for each of two filters (fields B and C), the proper motions were obtained from the weighted mean of the two measurements. For field B, we linked F814W (2001) with

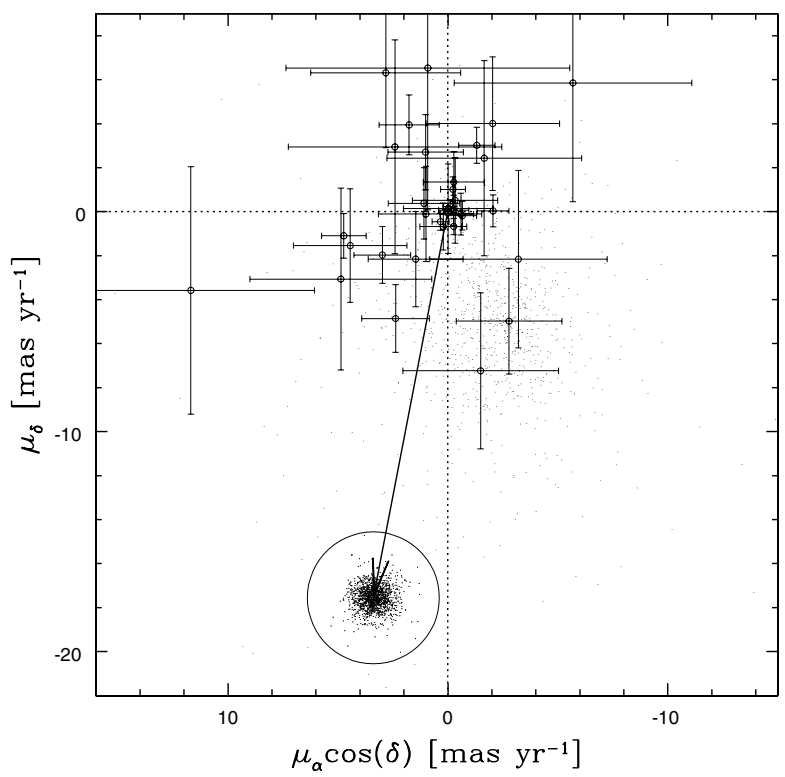

Fig. 2. Vector-point diagram of the proper motions in equatorial coordinates, combining all the fields. Open circles with error bars represent the selected reference galaxies. Dots represent the stars; a circle at $3 \mathrm{mas} / \mathrm{yr}$ separates cluster members from field objects. The arrow is the adopted absolute proper motion of NGC 6397.

F814W (1994) and also F606W (2001) with F606W (1996); in field C we combined $F 814 W$ (2001) with $F 814 W$ (1996), and also $F 606 W(2000)$ with $F 606 W(1996)$. In field D we matched F814W (2001) with F814W (1995).

A visual inspection of the images reveals many background galaxies. Since 33 of these have a point-like nucleus, we used our ePSF-fitting procedure to measure their positions. As was to be expected, the errors found from multiple measurements of galaxy positions are much larger than those that are typical of stars, and they are a strong function of the galaxy morphology. Nevertheless, those galaxies that give good positions provide an ideal reference point for the measurement of the absolute proper motions of stars.

Figure 2 shows the vector point diagrams for the three fields B, C, and D combined. Small points show the proper motions of the stars, and open circles show the motions measured for the selected galaxies. For each galaxy we also show the proper-motion error bar. The axes are parallel to right ascension and declination. Cluster members were used to compute the transformations and to derive the relative proper motions (see Paper I for details). For the pixel scale we adopted the number given by Anderson \& King (2003), and for the orientations we used information in the HST image headers. Cluster members constitute the concentrated distribution of points at the lower left. The separation between field stars and cluster stars is very well defined. Finally, the zero point of the figure was placed at the weighted mean motion of the galaxies; this converts all the motions to absolute. The centroid proper motions of the cluster stars are in good agreement for the three fields, as is shown in Table 2.

Taking the weighted mean of the positions of the whole sample of 33 galaxies as the zero point of the motion, we find for NGC 6397 an absolute proper motion, in the J2000.0 system, of

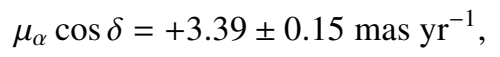

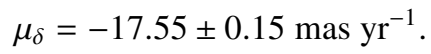


Table 2. Summary of absolute motion (in [mas/yr]) for each field. The number of background galaxies used to compute the reference point is also indicated.

\begin{tabular}{cccccc}
\hline \hline field & $\mu_{\alpha} \cos \delta$ & $\sigma_{\mu_{\alpha} \cos \delta}$ & $\mu_{\delta}$ & $\sigma_{\mu_{\delta}}$ & $N_{\text {gal }}$ \\
\hline $\mathrm{B}$ & +3.33 & 0.17 & -17.53 & 0.17 & 12 \\
$\mathrm{C}$ & +3.14 & 0.73 & -17.18 & 0.73 & 8 \\
$\mathrm{D}$ & +3.75 & 0.40 & -17.81 & 0.40 & 13 \\
\hline average & +3.39 & 0.15 & -17.55 & 0.15 & 33 \\
\hline
\end{tabular}

In Galactic coordinates this corresponds to

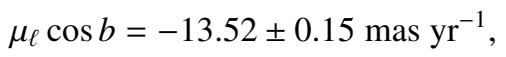

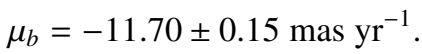

The dominant source of error is the uncertainty in the zero point given by the galaxies.

We note that the uncertainties of the results for the individual fields are quite different. This in turn rests on the wide range of the measurement errors for the individual galaxies, as indicated by the lengths of the error bars in Fig. 2. Nearly all of the weight comes from the few galaxies that have the best measurements; the majority of these are in Field B. This shows the importance of finding well-exposed galaxies with sharp centers.

These values are in marginal agreement with those found by Cudworth \& Hanson (1993), which give a proper motion that is $\sim 13 \%$ smaller. However, their values were based on a correction from relative to absolute proper motion that was obtained from a Galactic model, and their procedure seems to provide an analogous discrepancy ( $20 \%$ smaller) in the case of M 4 (Cudworth $\&$ Rees 1990), when compared with more recent direct determinations of its absolute proper motion (Dinescu et al. 1999; and Paper I).

\subsection{FLAMES+GIRAFFE/VLT: radial velocities}

With the aim of measuring the dispersion of radial velocities in NGC 6397, in order to estimate the distance of the cluster by comparing with the dispersion of proper motions (see Bedin et al. 2003b for a brief description of the method), we collected spectra of 1508 stars at the UT2-VLT telescope in May 2003 with the FLAMES/GIRAFFE spectrograph (proposal No. 71.D-0076). In the present paper we use these spectra to measure the mean radial velocity of the cluster. Observations were performed in the MEDUSA mode with simultaneous calibration, which allows simultaneously obtaining about 130 spectra of objects over a field of view of 25 arcmin in diameter. The high-resolution set-up H9 (now called H9B) was used; this setup allows a spectral resolution $R=26000$ in the 5140-5350 range. This spectral region is well-suited for radial-velocity measurements because of the large number of metallic lines and the absence of $\mathrm{H}$ features. It also includes the $\mathrm{Mg}$ triplet, which is particularly important in radial-velocity determinations for metal-poor stars like those of NGC $6397([\mathrm{Fe} / \mathrm{H}] \sim-2.0)$, because it is the strongest feature also clearly visible in low $S / N$ spectra.

The target stars for radial velocities were primarily selected on the basis of their position in the color magnitude diagram (CMD). The primary criterion used was to select stars along the red giant branch (RGB), the subgiant branch (SGB), and the main sequence (MS) down to $V=17.5$, on a narrow stripe 0.08 mag wide in color. This allowed us to drastically limit the contamination from field stars. Around the turn-off the stars of
NGC 6397 have a color that is 0.2 mag bluer than that of the contaminating stars of the Galactic disk. Moreover, the evolved stars in NGC 6397 occupy a position in the CMD that is well above the location of the nearby disk stars. By analyzing in an outer field, far from the cluster center, a stripe in the CMD of a similar size to our selection strip, we estimated that on average only 12 stars should be non-members, i.e., a smaller contamination than $1 \%$. (Furthermore, our radial velocities showed that stars in this part of the CMD are non-members.) This left virtually no contamination in the sample.

Data were automatically pre-reduced using the GIRAFFE pipeline GIRBLDRS ${ }^{1}$, in which the spectra have been de-biased, flat-field corrected, extracted, and wavelength-calibrated, using both prior and simultaneous calibration-lamp spectra. The resulting spectra have a dispersion of $0.05 \AA /$ pixel. A sky correction was applied to each stellar spectrum by using the IRAF sarith subroutine for subtraction of the average of the sky spectra that were observed simultaneously (same FLAMES plate) with the stars.

Radial velocities were obtained from the IRAF fxcor crosscorrelation subroutine. Stellar spectra were cross-correlated with a synthetic template calculated by SPECTRUM ${ }^{2}$, for the mean temperature, $\log g$, and metallicity appropriate for each observed star. The accuracy of this template then was tested on a solar spectrum obtained with the UVES spectrograph; the systematic error is less than $50 \mathrm{~m} \mathrm{~s}^{-1}$.

From multiple observations of 81 stars it was also possible to make a direct estimate of the errors. For stars of magnitude $V=14,15,16,17$, we find errors of $0.2,0.3,0.7$, and $1.4 \mathrm{~km} \mathrm{~s}^{-1}$, respectively. We also searched for possible systematic errors due to misalignment of the fibers or to the difference in the illumination of the simultaneous calibration fibers and the fibers used for the stars ${ }^{3}$. To this end we reduced the daytime wavelengthcalibration plates (the plates where all fibers are pointed at the wavelength-calibration ThAr lamp) using the same procedure that we used for our stellar plates. Then we measured the velocity shift of the fibers, using as a template spectrum the same ThAr template that was used for the wavelength calibration. As expected, the average radial velocity obtained from the spectra from the five simultaneus calibration fibers is zero, within the errors. By contrast, the radial velocities from the spectra from the remaining fibers show a systematic shift, different for each fiber, probably due to their incorrect alignment at the entrance of the spectrograph. For the purposes of the present paper, it is sufficient to consider the mean systematic shift in radial velocity. We obtained for the two positioner plates Medusa 1 and Medusa 2:

$\Delta V_{\text {rad }}$ [Medusa 1, NGC 6397] $=-0.175 \pm 0.009 \mathrm{~km} \mathrm{~s}^{-1}$, $\Delta V_{\text {rad }}$ [Medusa 2, NGC 6397] $=-0.225 \pm 0.008 \mathrm{~km} \mathrm{~s}^{-1}$,

and the mean value is

$\Delta V_{\mathrm{rad}}=-0.200 \pm 0.025 \mathrm{~km} \mathrm{~s}^{-1}$,

which must be subtracted from the cluster mean radial velocity.

As a further test we measured the systematic error affecting similar observations of another cluster, M 4, which were collected a month later. In this way we can test the time stability of

\footnotetext{
1 Blecha et al. (2000). See http://girbldrs . sourceforge.net/, for GIRAFFE pipeline, software and documentation.

2 The LTE spectral synthesis program freely distributed by Richard O. Gray. Program and documentation available at www . phys.appstate.edu/spectrum/spectrum.html

3 See the GIRAFFE pipeline FAQ web page, http:// girbldrs. sourceforge.net/FAQ/index . cgi
} 


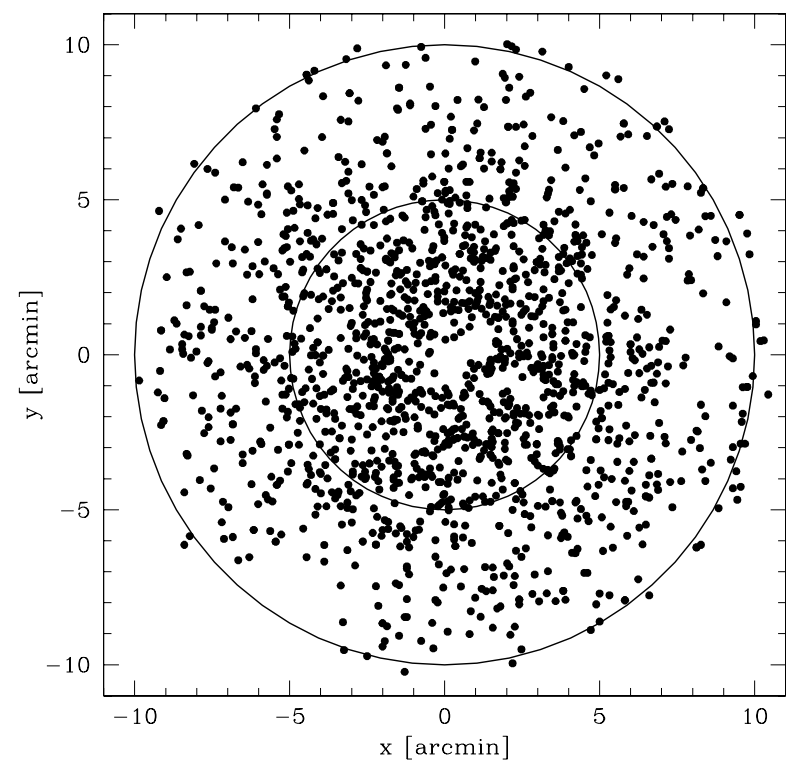

Fig. 3. Spatial distribution of stars with radial velocity measurement. The circles indicate the radial distances of 5 and 10 arc min from the center of the cluster.

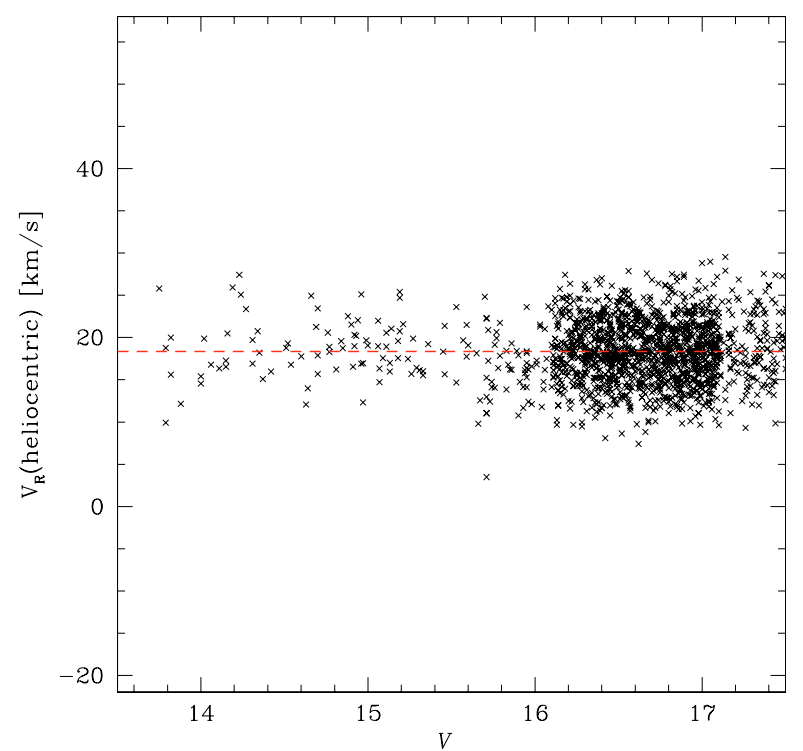

Fig. 4. Radial velocities of the observed sample of stars as a function of magnitude.

the spectrograph by seeing whether these numbers remain constant. We obtained in an analogous way for the data-set of M4 the mean systematic shift in radial velocity, for the two positioner plates Medusa 1 and Medusa 2:

$$
\begin{aligned}
& \Delta V_{\text {rad }}\left[\text { Medusa 1, M 4] }=-0.176 \pm 0.008 \mathrm{~km} \mathrm{~s}^{-1}\right. \\
& \Delta V_{\text {rad }}\left[\text { Medusa 2, M 4] }=-0.236 \pm 0.008 \mathrm{~km} \mathrm{~s}^{-1}\right.
\end{aligned}
$$

These values agree very well with the previous ones. From these results we can assert that GIRAFFE is stable, at least for a period of about a month.

The spatial distribution of our stars, and their heliocentric radial velocities as a function of visual magnitude, are shown in Figs. 3 and 4.
The not-weighted average radial velocity ${ }^{4}$, corrected for the systematic shift discussed previously and clipped for outliers more than $15 \mathrm{~km} \mathrm{~s}^{-1}$ from the mean, is

$V_{\text {rad }}=18.36 \pm 0.09( \pm 0.03) \mathrm{km} \mathrm{s}^{-1}$.

The two errors that are given are first the random error and then the uncertainty in the correction for the systematic shift. This mean comes from 1486 stars (22 were rejected).

However, there are other effects of the same size that afflict spectroscopic measurements of radial velocities, such as the blue-shift of convective bubbles in giant stars or gravitational redshift in dwarf stars (Dravins et al. 1999). Since we have observed both giant and dwarf stars, we add a global systematic error of $0.10 \mathrm{~km} \mathrm{~s}^{-1}$ to our uncertainties. Finally,

$V_{\mathrm{rad}}=18.36 \pm 0.09( \pm 0.10) \mathrm{km} \mathrm{s}^{-1}$.

[When we analyze the cluster internal dynamics, we will carefully correct for all these effects, and also each fiber will be corrected individually for the calibration bias described above.]

Our heliocentric average radial velocity for NGC 6397 is within $\sim 1.3 \sigma$ of the Da Costa et al. (1977) value of $V_{\text {rad }}=$ $19.8 \pm 1.1 \mathrm{~km} \mathrm{~s}^{-1}$, within $\sim 2.1 \sigma$ of the Dubath et al. (1997) value of $V_{\text {rad }}=15.1 \pm 1.6 \mathrm{~km} \mathrm{~s}^{-1}$, but there is a large disagreement $(\sim 5 \sigma)$ with the $V_{\mathrm{rad}}=18.80 \pm 0.10 \mathrm{~km} \mathrm{~s}^{-1}$ given by Meylan $\&$ Mayor (1991).

Dubath et al. measured the radial velocity on an integratedlight echelle spectrum of the cluster. Kinematical measurements of this kind of spectra are known to be affected by strong observational biases due to small-number statistics and strong luminosity weighting (of a few red giants). As clearly demonstrated by Zaggia et al. (1992, 1993), these biases influence both velocity dispersion and radial velocity: systematic offsets can be as large as $1 \times$ the velocity dispersion itself. Therefore it is not surprising to find a discrepancy between the integrated-light radial velocity and the average radial velocity of individual stars.

It is more problematic to explain the discrepancy with the Meylan \& Mayor (1991) radial velocity, considering that errors in their single velocities are similar to ours, even though their sample is only $\sim 1 / 12$ the size of our. Their measure is based on CORAVEL spectrometer observations of 127 RGB and SGB stars. A possible source of systematic error could possibly be in a spectral mismatch of the CORAVEL mask with the lowmetallicity spectra of NGC 6397 stars, as Meylan \& Mayor indeed mention in their paper. Another source of systematic error could be the presence of unrecognized field stars which could bias the mean radial velocity, considering the low velocity of NGC 6397. Meylan \& Mayor observed mainly RGB stars in NGC 6397, while the majority of our targets are turn-off and MS stars, which should be relatively free of field contamination. Unfortunately a direct comparison of the radial velocities of the two datasets is not possible.

Considering the work done to limit all possible systematics in the GIRAFFE spectra, we believe that with a final systematic error $<0.1 \mathrm{~km} \mathrm{~s}^{-1}$ and the large-number statistics of nearly 1600 radial velocities, our mean radial velocity is a significant improvement over previous measurements.

\footnotetext{
${ }^{4}$ Note that the choice of weights and the calculation of the uncertainty in the mean velocity depend both on the velocity dispersion of the cluster and on the uncertainties of the individual velocities (see Pryor \& Meylan 1993). A much more careful analysis will be done in our future work on the internal dynamics.
} 
Table 3. Input conditions for orbit calculation. Space velocities in $\mathrm{km} \mathrm{s}^{-1}$.

\begin{tabular}{ccccc}
\hline \hline \multicolumn{5}{c}{ Distance $1.8 \mathrm{kpc}$} \\
$U$ & $V$ & $W=Z$ & $\Pi$ & $\Theta$ \\
$34 \pm 5$ & $-92 \pm 11$ & $-94 \pm 10$ & $22 \pm 5$ & $131 \pm 11$ \\
\hline \multicolumn{5}{c}{ Distance $2.0 \mathrm{kpc}$} \\
$U$ & $V$ & $W=Z$ & $\Pi$ & $\Theta$ \\
$41 \pm 5$ & $-103 \pm 11$ & $-105 \pm 10$ & $28 \pm 5$ & $121 \pm 11$ \\
\hline \multicolumn{5}{c}{ Distance $2.2 \mathrm{kpc}$} \\
$U$ & $V$ & $W=Z$ & $\Pi$ & $\Theta$ \\
$48 \pm 5$ & $-114 \pm 11$ & $-116 \pm 10$ & $35 \pm 5$ & $111 \pm 11$ \\
\hline \multicolumn{5}{c}{ Distance $2.4 \mathrm{kpc}$} \\
$U$ & $V$ & $W=Z$ & $\Pi$ & $\Theta$ \\
$55 \pm 5$ & $-125 \pm 11$ & $-126 \pm 10$ & $42 \pm 5$ & $101 \pm 11$ \\
\hline \multicolumn{5}{c}{}
\end{tabular}

\section{Calculation of the orbital parameters}

Our cluster radial velocity and absolute proper motion allow us to derive the three velocity components of NGC 6397 and estimate its Galactic orbit. The new orbit should help us to trace back the dynamical history of the cluster, clarify how close it goes to the Galactic center, and assess the possible impact of the motion on its internal dynamics and its mass function.

Orbit integration for a globular cluster requires adopting a model of the potential of the Milky Way. We chose that of Allen \& Santillan (1991), which was constructed to fit an assumed rotation curve and a distance and velocity of the Sun with respect to the Galactic center. The potential is time-independent, so clearly a crude approximation, because a significant variation of the Galactic potential is expected over a typical globularcluster lifetime. Nevertheless, it is reasonable to believe that the Galactic potential has not changed much in the last few Gyr, so that the derived parameters for the orbit of NGC 6397, such as the apo- and perigalacticon, can be considered to be reasonable estimates.

The Galactic potential of Allen \& Santillan (1991) assumes density distributions in the bulge, disk, and halo components. This gravitational potential is time-independent, axisymmetric, fully analytic, and mathematically very simple. It has already been used to derive the Galactic orbits of nearby stars (Allen \& Martos 1986), open clusters (Carraro \& Chiosi 1994), and disk and halo GCs (Odenkirchen \& Brosche 1992). The initial conditions are given in Table 3 for different cluster distances, assuming $R_{\circ}=8.5 \mathrm{kpc}$, and the value of $V_{\circ} / R_{\circ}$ derived in Paper I.

The integration routine is a fifteenth-order symmetric, simplectic Runge-Kutta, using the Radau scheme (Everhart 1985); it guarantees energy and momentum conservation at the levels of $10^{-12}$ and $10^{-9}$, respectively, over the whole orbit integration. The orbits have been integrated back in time for 5 Gyr and are shown in Fig. 5.

The orbital parameters are summarized in Table 4, where Col. (1) lists the adopted cluster heliocentric distance, Col. (2) the $z$-component of the angular momentum, Col. (3) the total energy, Col. (4) the orbital period (for radial motion and for vertical motion), Cols. (5) and (6) the apo- and peri-center of the orbit, Col. (7) the maximum vertical distance the cluster reaches and Col. (8) the eccentricity, defined as $\left(R_{\mathrm{a}}-R_{\mathrm{p}}\right) /\left(R_{\mathrm{a}}+R_{\mathrm{p}}\right)$. The orbit parameters that we found do not differ much from those published by Dauphole et al. (1996) and by Dinescu et al. (1999), which means basically that the Galactic model we adopt in this paper is consistent with those used by the previous authors. Indeed, Dauphole et al. (1996) used an earlier version (Allen \& Martos 1986) of the Galactic model we use here
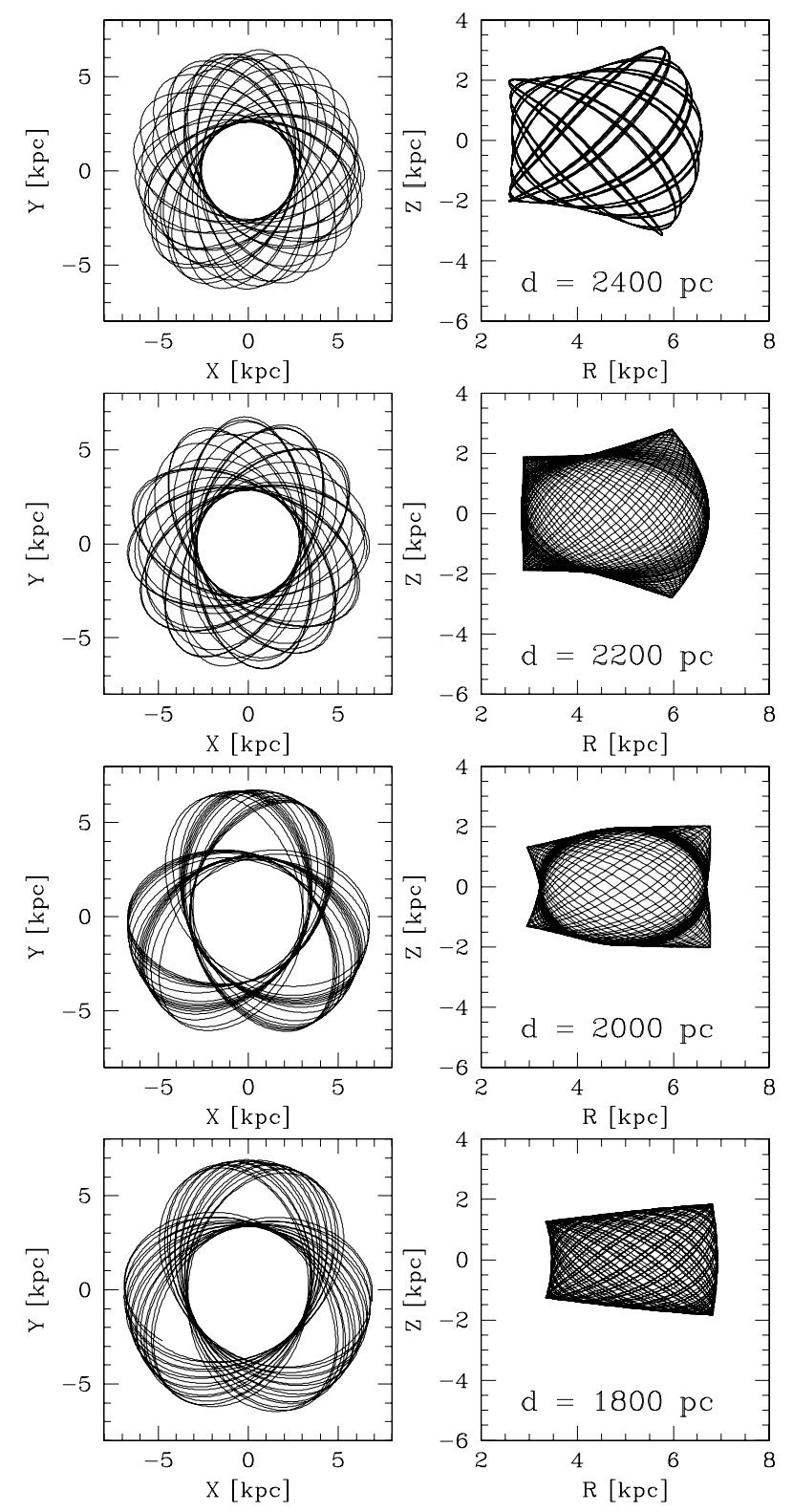

Fig. 5. Calculated orbits for assumed distances 1.8, 2.0, 2.2, and $2.4 \mathrm{kpc}$.

(Allen \& Santillan 1991). In any case, our newly derived space velocities make our results an improvement over previous investigations. In particular, this is the first time that proper motions calibrated directly against extragalactic sources have been used in studying the orbit of NGC 6397.

Independent of the initial conditions chosen, the orbit of NGC 6397 has a boxy nature. The cluster oscillates rapidly through a dense part of the Galactic plane, only a few kiloparsecs from the Galactic center. This kind of orbit makes NGC 6397 very vulnerable to tidal shocks, with a consequent preferential loss of low-mass stars, which, as first recognized by Piotto et al. (1997, see their detailed discussion), is the likely cause of its anomalously flat mass function.

As the heliocentric distance increases (from bottom to top panel) the cluster tends to have a longer period, to reach greater heights above the Galactic plane and to show a larger epicyclic amplitude, dipping closer to the Galactic center. The assumed distance has a complicated effect on tidal shocks, which work over time to flatten the mass function of the cluster. Tidal shocks 
Table 4. Orbit parameters, for the four different distances. Unit description: $d[\mathrm{kpc}], L_{\mathrm{z}}\left[\mathrm{kpc} \mathrm{km} \mathrm{s}{ }^{-1}\right], E_{\mathrm{tot}}\left[10 \times \mathrm{km}^{2} \mathrm{~s}^{-2}\right], P[\mathrm{Myr}], R_{\mathrm{a}}[\mathrm{kpc}]$, $R_{\mathrm{p}}[\mathrm{kpc}], z_{\max }[\mathrm{kpc}], e$ [pure number].

\begin{tabular}{cccccccc}
\hline \hline$d$ & $L_{\mathrm{z}}$ & $E_{\mathrm{tot}}$ & $P$ & $R_{\mathrm{a}}$ & $R_{\mathrm{p}}$ & $z_{\max }$ & $e$ \\
$(1)$ & $(2)$ & $(3)$ & $(4)$ & $(5)$ & $(6)$ & $(7)$ & $(8)$ \\
\hline 1.8 & 933 & -10203 & 112 & 6.92 & 3.34 & 1.84 & 0.35 \\
2.0 & 846 & -9899 & 102 & 6.78 & 2.94 & 2.02 & 0.39 \\
2.2 & 766 & -9634 & 99 & 6.74 & 2.83 & 2.80 & 0.41 \\
2.4 & 693 & -9424 & 93 & 6.59 & 2.57 & 3.11 & 0.44 \\
\hline
\end{tabular}

occur three times in each orbital period: at perigalactic passage and at each passage through the Galactic plane. For a greater assumed distance the cluster is shocked more frequently, and the shocks due to the Galactic center are stronger, but the shocks due to the plane occur at higher cluster velocity and are thus less effective.

Note that the major source of uncertainty in the initial conditions is our ignorance of the cluster's distance. A more accurate distance determination is in progress, based on the same observing material that is presented in this paper. It will be a fundamental step in our understanding of the motion of NGC 6397.

Acknowledgements. G.P. and A.M. acknowledge the support by the MIUR under the program PRIN2003. I.R.K. and J.A. were supported by STScI grants GO-8656 and AR-8736. S.Z. was supported by the MIUR program PRIN2004. We thank the referee, Dr. C. Pryor, for a careful reading of the manuscript and for many useful comments.

\section{References}

Allen, C., \& Martos, M. A. 1986, Mex. Astron. Astrofis., 13, 137

Allen, C., \& Santillan, A. 1991, Mex. Astron. Astrofis., 22, 255

Anderson, J., \& King, I. R. 1999, PASP, 111, 1095

Anderson, J., \& King, I. R. 2000, PASP, 112, 1360

Anderson, J., \& King, I. R. 2003, PASP, 115, 113

Bedin, L. R., Piotto, G., Anderson, J., \& King, I. R. 2003, AJ, 126, 247 (Paper I)

Bedin, L. R., Piotto, G., Anderson, J., \& King, I. R. 2003b, New Horizons in Globular Cluster Astronomy, ed. G. Piotto, G. Meylan, S. G. Djorgovski \& M. Riello, ASP Conf. Ser., 296, 360

Blecha, A., Cayatte, V., North, P., Royer, F., \& Simond, G. 2000, Proc. SPIE, 4008, 467

Carraro, G., \& Chiosi, C. 1994, A\&A, 288, 751

Cudworth, K. M., \& Rees, R. 1990, AJ, 99, 1491

Cudworth, K. M., \& Hanson, R. B. 1993, AJ, 105, 168

Da Costa, G. S., Freeman, K. C., Kalnajs, A. J., Rodgers, A. W., \& Stapinski, T. E. 1977, AJ, 82, 810

Dauphole, B., Geffert, M., Colin, J., et al. 1996, A\&A, 313, 119

Dinescu, D. I., van Altena, W. F., \& Girard, T. M. 1999, AJ, 117, 277

Dravins, D., Lindegren, L., \& Madsen, S. 1999, A\&A, 348, 1040

Dubath, P., Meylan, G., \& Mayor, M. 1997, A\&A, 324, 505

Everhart, E. 1985, in Dynamics of Comets: Their Origin and Evolution, ed. A. Carusi \& G. B. Valsecchi (Dordrecht: Reidel), Proc. IAU Coll., 83, 185

Holtzman, J. A., Burrows, C. J., Casertano, S., et al. 1995, PASP, 107, 1065

Meylan, G., \& Mayor, M. 1991, A\&A, 250, 113

Odenkirchen, M., \& Brosche, P. 1992, Astron. Nachr., 313, 69

Pasquini, L., et al. 2002, Msngr, 110, 1

Piotto, G., Cool, A. M., \& King, I. R. 1997 AJ, 113, 1345

Pryor, C., \& Meylan, G. 1993, in Structure and Dynamics of Globular Clusters, ed. S. G. Djorgovski, \& G. Meylan, ASP Conf. Ser., 50, 357

Zaggia, S. R., Capaccioli, M., Piotto, G., \& Stiavelli, M. 1992, A\&A, 258, 302

Zaggia, S. R., Capaccioli, M., \& Piotto, G. 1993, A\&A, 278, 415 